\title{
ESTUDO EXPERIMENTAL DA CONVECÇÃO NATURAL DO CALOR EM UMA PLACA DE GELO RETANGULAR
}

Renato Letizia Garcia-renato-garcia@uergs.edu.br

Universidade Estadual do Rio Grande do Sul - Unidade de Porto Alegre

Av. Bento Gonçalves, 8855 - bairro Agronomia

CEP 91450-000 - Porto Alegre - RS

RoseliKuhn-roseli-kuhn@uergs.edu.br

Universidade Estadual do Rio Grande do Sul - Unidade de Porto Alegre Av. Bento Gonçalves, 8855 - bairro Agronomia

CEP 91450-000 - Porto Alegre - RS

Vanessa Kristine de Oliveira Schmidt-vanessakschmidt@hotmail.com

Universidade Estadual do Rio Grande do Sul - Unidade de Porto Alegre

Av. Bento Gonçalves, 8855 - bairro Agronomia

CEP 91450-000 - Porto Alegre - RS

Rafael AislanAmaral - rafael.amaral@ufrgs.edu.br

Universidade Federal do Rio Grande do Sul-Campus Litoral Norte

Rodovia RS 030, km 92 - bairro Emboaba

CEP 95590-000 - Tramandai - RS

Edson Abel dos Santos Chiaramonte-edsonchiaramonte@unipampa.edu.br

Universidade Federal do Pampa - Campus Bagé

Av. Maria Anunciação Gomes de Godoy, 1650-bairro Malafaia

CEP 96413-172 - Bagé - RS

Resumo: Nesse artigo, o processo de fusão de uma placa de gelo retangular exposta ao ar ambiente é utilizado para o estudo experimental dos mecanismos de transferência de calor e massa, e dos números adimensionais envolvidos na convecção natural. A proposta de investigar a influência do posicionamento espacial da placa na fusão do gelo é empregada como forma de aguçar a curiosidade e o interesse do aluno no aprendizado de fenômenos associados à transferência de calor e massa. Conforme detalhado no texto, convecção e radiação do calor, assim como a condensação da umidade do ar, são os mecanismos de transferência de calor relevantes no derretimento do gelo, sendo empregado um modelo matemático que calcula a parcela de calor associada a esses mecanismos. Os valores teóricos são confrontados com os dados experimentais e os resultados quantitativos obtidos foram satisfatórios, confirmando as previsões teóricas relativas a essa influência.

Palavras-chave: Primeira palavra. Segunda palavra. Terceira palavra (máximo de 5). 


\section{INTRODUÇÃO}

O estudo da transferência de calor nos cursos de graduação se inicia, geralmente, pela descrição dos três mecanismos responsáveis por esse processo - radiação, condução e convecção - e de suas respectivas equações. A convecção do calor, que pode ser descrita como a troca térmica produzida pela movimentação de um fluido sobre uma interface sólida, é regida pela geometria da interface, características do fluido e do escoamento desse fluido sobre essa interface (HOLMAN, 1983).

No intuito de avaliar a influência dos diversos fatores na convecção de calor, os livros didáticos que abordam esse assunto dedicam capítulos específicos a situações distintas envolvendo esse mecanismo de troca térmica (INCROPERA; DE WITT, 2008). Esses capítulos apresentam inúmeras equações e correlações empíricas, envolvendo números adimensionais e variáveis associadas à geometria da interface, dificultando a interpretação dos fenômenos físicos a partir de sua formulação matemática (ÇENGEL; GHAJAR, 2012). O aluno de graduação tende a ficar ainda mais apreensivo e desestimulado na medida em que é solicitado a resolver problemas a partir da aplicação de fórmulas e da eventual necessidade de memorização dessas fórmulas para realizar as provas da disciplina que trata desse tema (MARCZAK et al, 2003).

Nesse artigo é apresentada uma proposta experimental para o estudo do processo de convecção, baseado num ensaio de fusão de uma placa retangular de gelo, a partir da formulação da seguinte pergunta: "A placa de gelo irá derreter mais rapidamente em função de seu posicionamento espacial?"

No ensaio proposto, uma placa de gelo de pequena espessura, que pode ser obtida facilmente pelo congelamento de água em uma fôrma metálica (do tipo utilizada para assar bolos), é fixada a um suporte e suspensa no ar de duas maneiras distintas: o comprimento (maior dimensão) na direção vertical e a largura (menor dimensão) na direção vertical.

O tipo de questão formulada desperta o interesse da maioria das pessoas, mesmo daquelas que não possuem formação acadêmica ou que não são afeitas às ciências exatas, e todos tendem a emitir uma opinião. Esse interesse, aliado a uma certa curiosidade, decorre do fato de ser uma questão simples e de aplicação no cotidiano e que irá estimular o aluno da graduação a buscar uma melhor compreensão da convecção natural do calor, na medida em que ele identifica a possibilidade de emprego das equações apresentadas em aula em uma situação real. $\mathrm{O}$ experimento proposto envolve a realização de um ensaio de fácil execução e com emprego de equipamento de baixo custo, viabilizando tal experimento como uma ferramenta pedagógica acessível para o ensino de Fenômenos de Transporte.

\section{FUNDAMENTOS TEÓRICOS}

A Lei de resfriamento de Newton, proposta em 1701, nos diz que a taxa da variação da temperatura de um corpo $(d T / d t)$ é proporcional à diferença entre a temperatura desse corpo e do ambiente que o circunda, sendo expressa através da equação:

$$
\frac{d T}{d t}=k .\left(T-T_{a m b}\right)
$$

na qual $\mathbf{T}$ é a temperatura do corpo em um instante qualquer, $\mathbf{T}_{\mathbf{a m b}}$ é a temperatura ambiente, e k é uma constante de proporcionalidade. Estudos posteriores de transferência do calor permitiram caracterizar três mecanismos de transporte de energia térmica: condução, radiação e convecção. A taxa de transferência de calor por convecção, qconv., é dada pela seguinte equação: 


$$
q_{c o n v}=h \cdot A \cdot\left(T-T_{a m b}\right)
$$

na qual $\mathbf{A}$ é a área do corpo exposta ao ambiente, e $\mathbf{h}$ é o coeficiente de transferência convectivo de calor. A radiação de calor também se faz presente quando se analisa o resfriamento de um corpo aquecido, sendo que a taxa de calor, $\mathbf{q}_{\text {rad., é dada pela equação: }}$

$$
q_{\text {rad }}=\epsilon \cdot \sigma \cdot A \cdot\left(T^{4}-T_{a m b}^{4}\right)
$$

na qual $\varepsilon$ é a emissividade da superfície desse corpo, $\boldsymbol{\sigma}$ é a constante de Stefan-Boltzmann, cujo valor é $5,67 \cdot 10^{-8} \mathrm{~W} /\left(\mathrm{m}^{2} \cdot \mathrm{K}^{4}\right)$.

\subsection{O balanço térmico na placa de gelo}

Nos problemas reais de resfriamento de um corpo ao ambiente é necessário, em muitas situações, considerar a contribuição dos mecanismos de radiação e convecção do calor. No modelo proposto para análise da fusão da placa de gelo, as duas contribuições serão avaliadas, de modo que o calor absorvido pela placa será o produto da massa de gelo derretida, $\mathbf{m}_{\mathbf{a g} .}$, pelo calor latente de fusão do gelo, $\mathbf{L}_{\mathbf{f}}$, segundo a expressão:

$$
m_{a g .} \cdot L_{f}=h \cdot A \cdot\left(T_{a m b}-T\right) \cdot d t+\epsilon \cdot A \cdot\left(T_{a m b}^{4}-T^{4}\right) \cdot d t
$$

A exposição de uma superfície fria ao ar ambiente tenderá a promover a condensação da umidade do ar sobre essa superfície, como ocorre na superfície externa de copos ou recipientes com bebidas geladas. Essa condensação de umidade, no caso da placa de gelo, irá aumentar a massa de água que escorre da placa; e, simultaneamente, irá transferir uma parcela adicional de calor para esse objeto sólido, além das parcelas de calor radiativo e convectivo que são absorvidas em sua superfície. Assim, a massa de gelo derretida corresponde à massa de água proveniente da placa, $\mathbf{m}_{\mathbf{a g}}$, menos a parcela de condensação do vapor de água proveniente do ar atmosférico, $\mathbf{m}_{\text {cond. }}$. Por sua vez, o calor transferido no processo de condensação da umidade é igual ao produto da massa de vapor de água condensado pela calor latente de vaporização da água, $\mathbf{L}_{\mathbf{v a p}}$., resultando na equação:

$$
\left(m_{a g}-m_{\text {cond. }}\right) \cdot L_{f}=h \cdot A \cdot\left(T_{a m b}-T\right) \cdot d t+\epsilon \cdot A \cdot\left(T_{a m b}^{4}-T^{4}\right) \cdot d t+m_{c o n d .} \cdot L_{v a p} .
$$

Essa parcela de água condensada é proporcional à taxa de transferência convectiva de massa, $\mathbf{h}_{\mathbf{m}}$, cuja expressão matemática é:

$$
m_{\text {cond }}=h_{m} \cdot A \cdot\left(\rho_{v, a m b}-\rho_{v, s}\right) \cdot d t,
$$

na qual $\boldsymbol{\rho}_{\mathbf{v}, \mathbf{a m b}}$ e $\boldsymbol{\rho}_{\mathbf{v}, \mathbf{s}}$, correspondem, respectivamente, à concentração do vapor de água no ar atmosférico e junto à superfície da placa. Cabe aqui ressaltar a analogia entre as equações (2) e (6), associadas às taxas de transferência convectiva, respectivamente, de calor e de massa. Isso nos leva a concluir que o processo de transporte de massa e de calor é similar, como será visto nas seções subsequentes.

\subsection{Números adimensionais e correlações empíricas na convecção natural}

A simplicidade da equação (1), proposta por Newton para representar o processo de resfriamento de um corpo sólido, é mantida na expressão matemática utilizada na equação (2), 
que quantifica a taxa de transferência convectiva de calor. Contudo, a determinação da taxa de transferência convectiva de calor não constitui, de modo geral, uma tarefa simples; e, adicionalmente, os valores teóricos estimados para o coeficiente $\mathbf{h}$ podem apresentar desvios significativos quando confrontados com os valores verificados na prática (BEJAN, 1996). A importância de avaliar o calor transferido em processos convectivos em sistemas físicos reais resultou na produção de inúmeras pesquisas teóricas e experimentais sobre o assunto, envolvendo objetos sólidos nas mais variadas geometrias e diferentes regimes de escoamento de fluidos junto à uma interface sólida.

Nos estudos desenvolvidos no campo da mecânica dos fluidos, tendo em vista a dificuldade da resolução de problemas reais a partir de metodologias baseadas apenas no emprego de equações diferenciais e integrais, foram elaborados métodos experimentais para estabelecer relações entre as variáveis de interesse. Dentro desse contexto surgiu uma técnica chamada análise dimensional, que é baseada no emprego de números adimensionais para estimar alguns coeficientes teóricos, como os coeficientes convectivos de transferência de calor e massa.

\subsection{Determinação do coeficiente convectivo de transferência de calor}

Grande parte dos números adimensionais podem ser caracterizados como uma razão entre duas forças opostas que atuam em um processo físico. Na convecção natural do calor, o número de Grashoff (Gr) é uma razão entre as forças empuxo e forças viscosas atuantes sobre o fluido junto a um objeto sólido (KAYS; CRAWFORD; WEIGHAND, 2004), sendo expresso na seguinte forma:

$$
G r=\frac{g \cdot \beta \cdot \Delta T \cdot L_{c}^{3}}{v^{2}}
$$

sendo $\boldsymbol{g}$ a aceleração da gravidade, $\boldsymbol{\beta}$ o coeficiente de expansão térmica do fluido, $\boldsymbol{\Delta T}$ a diferença entre a temperatura da placa de gelo e a temperatura do fluido, $\mathbf{L}_{\mathbf{c}} \mathbf{O}$ comprimento característico do objeto sólido (no caso, a placa de gelo), e $\boldsymbol{v}$ a difusividade cinemática do fluido. Cabe aqui ressaltar que, no cálculo do $\Delta \mathrm{T}$, a temperatura do fluido é a temperatura do ar ambiente, pois é a diferença entre a temperatura da placa e a temperatura ambiente que induz a movimentação do ar sobre a placa. Por sua vez, a difusividade cinemática e o coeficiente de expansão térmica são calculados com base na temperatura de filme, isto é, a temperatura média entre a placa de gelo e o ar ambiente; sendo que tal fato decorrente do pressuposto que existe uma camada de ar de espessura diminuta (filme) na qual há a transferência de calor entre a placa e o ambiente externo. O coeficiente de expansão térmica corresponde ao inverso da temperatura (absoluta) de filme. O comprimento característico da placa de gelo é a sua altura, tendo em vista que o escoamento do ar junto à placa ocorre na direção vertical.

O número de Nusselt $(\mathrm{Nu})$ para uma placa plana vertical é dado pela expressão:

$$
N u=0,59 .(G r . \operatorname{Pr})^{0,25}
$$

que estabelece uma correlação entre o número Nusselt com o de Grashoff e o de Prandtl (Pr); sendo este último a razão entre a difusividade térmica e a difusividade cinemática do fluido (no caso, o ar) na temperatura de filme. O número de Nusselt representa a razão entre a taxa de transferência de calor convectiva e condutiva no filme de fluido que reveste a superfície do objeto sólido (FOUST et al., 1982), sendo expresso através da seguinte fórmula matemática: 


$$
N u=\frac{h \cdot L_{c}}{k_{f}}
$$

sendo $\mathbf{k}_{\mathbf{f}}$ a condutividade térmica do fluido na temperatura de filme. As equações (7) a (9) possibilitam determinar o valor do coeficiente convectivo de transferência de calor a partir das características geométricas do objeto sólido e das propriedades termofísicas do fluido.

\subsection{Determinação do coeficiente convectivo de transferência de massa}

No processo de transferência convectiva de massa, num processo que ocorre em virtude da diferença de concentração de um componente de uma mistura, sem a movimentação forçada de fluido, o número de Grashoff é dado pela seguinte expressão:

$$
G r=\frac{g \cdot \Delta \rho \cdot L_{c}^{3}}{\rho \cdot v^{2}}
$$

sendo $\Delta \rho$ a diferença entre a concentração do fluido nos dois meios entre os quais o componente se transfere, e $\boldsymbol{\rho}$ a média dos valores das concentrações do fluido nesses dois meios. Inicialmente, para o experimento realizado, é preciso considerar a presença de uma película de água líquida revestindo a placa de gelo e, junto a ela, uma película de ar que se encontra na mesma temperatura da superfície da placa, $\mathrm{T}_{\mathrm{s}}$, isto é, $0^{\circ} \mathrm{C}$. $\mathrm{O}$ ar afastado da placa se encontra na temperatura ambiente e, consequentemente, há uma variação da concentração do ar entre a superfície da placa e um ponto afastado dela. Em ambas as situações admite-se que o ar comporta-se como gás ideal, sendo a concentração, em cada caso, calculada com o valor da respectiva temperatura.

O próximo passo é a determinação do número de Sherwood (Sh), cuja expressão é:

$$
S h=0,59 .(G r . S c)^{0,25}
$$

na qual Sc é o número de Schmidt. A similaridade entre as equações (11) e (8) deve-se ao fato de que ambas estão vinculadas a um processo de convecção natural em placa plana vertical. $\mathrm{O}$ número de Schmidt é a razão entre a difusividade do componente A numa mistura, $\mathbf{D}_{\mathbf{A B}}$, e a difusividade cinemática do fluido, $v$, o que nos leva à seguinte expressão:

$$
S c=\frac{v}{D_{A B}}
$$

sendo tais difusividades calculadas na temperatura de filme, isto é, a média entre a temperatura do ar ambiente e da placa de gelo. O número de Sherwood também está relacionado ao comprimento característico da placa de gelo através da fórmula:

$$
S h=\frac{h_{m} \cdot L_{c}}{D_{A B}}
$$

na qual $\mathbf{h}_{\mathbf{m}}$ é o coeficiente convectivo de transferência de massa.

A determinação do valor de $\mathrm{h}_{\mathrm{m}}$ é necessária para avaliar a taxa de condensação do vapor de água presente no ar ambiente sobre a superfície da placa de gelo, dada pela equação: 


\section{COBENCE de Educação em Engenharia da ABENGE}

$$
\dot{m}_{\text {cond }}=h_{m} \cdot A \cdot\left(\rho_{v, a m b}-\rho_{v, s}\right)
$$

sendo as pressões de vapor de água, junto à placa de gelo e no ar ambiente, respectiavamente, $\boldsymbol{\rho}_{\mathrm{v}, \mathrm{s}}$ e $\boldsymbol{\rho}_{\mathrm{v}, \mathbf{a m b}}$, calculadas admitindo que o vapor de água se comporta como gás ideal.

\section{METODOLOGIA}

O experimento proposto inicia-se pela obtenção de uma placa de gelo retangular, com o emprego de uma fôrma metálica, medindo $29 \mathrm{~cm}$ de comprimento e $19 \mathrm{~cm}$ de largura, na qual água é congelada com a prévia inserção de barbantes para facilitar a suspensão dessa placa em um suporte metálico na forma de "U" invertido (vide Figuras 1 e 2).

Figuras 1 e 2 - Ensaios realizados com a placa de gelo.

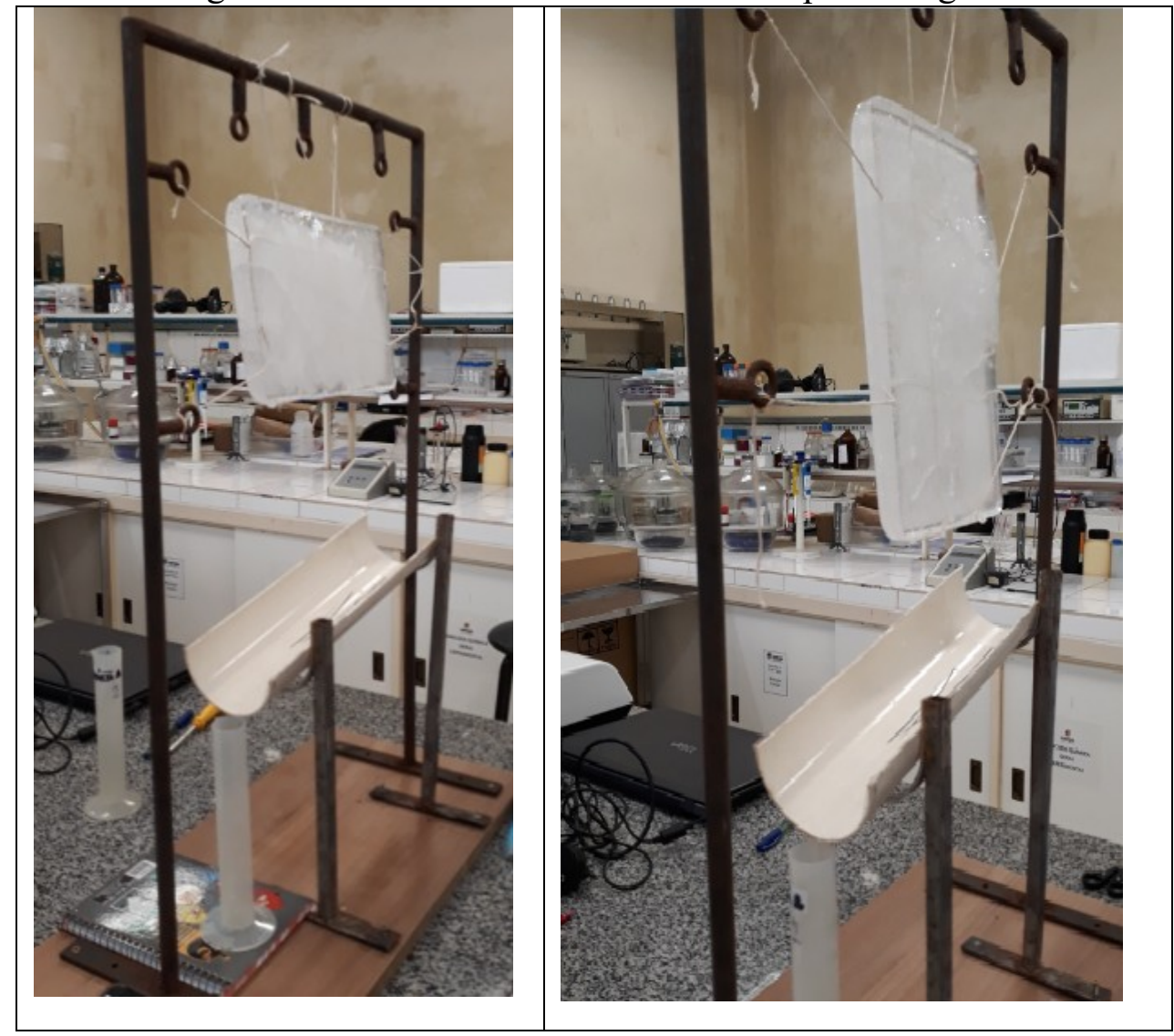

Fonte: autores

Foram realizados dois ensaios, num dos quais a altura da placa correspondia ao comprimento da fôrma; e, no outro, à sua largura. A temperatura e a umidade relativa do ar durante a realização dos ensaios foram de $23^{\circ} \mathrm{C}$ e $75 \%$. Em ambos os ensaios, a água proveniente do derretimento da placa foi coletada em uma proveta, e foi registrada a massa de água coletada a cada minuto.

Na Figura 3 é apresentado um gráfico relacionando a quantidade de água coletada na proveta durante a realização do ensaio no qual a altura da placa corresponde ao comprimento da fôrma metálica. Nesse gráfico, observa-se que, após decorrido algum tempo, a quantidade de água coletada na proveta não sofre variações significativas, até o final do experimento. Isso 
ocorre porque que a placa de gelo, no início do ensaio, está numa temperatura inferior a $0^{\circ} \mathrm{C}$, aumentando progressivamente sua temperatura ao longo do processo. Após atingir essa temperatura, o calor é transferido para a placa apenas na forma de calor latente, de modo que a quantidade de gelo derretida oscila levemente entre os intervalos. $\mathrm{O}$ ensaio descrito foi realizado durante 24 minutos; sendo que, após 7 minutos, não se verifica mais o aumento contínuo da massa de água derretida. Para fins comparativos, foi calculado o valor médio da massa de água coletada a cada minuto a partir desse instante, obtendo-se um valor de 7,20 gramas, que foi representado no gráfico através de uma linha laranja, de modo a destacar a oscilação dos dados observados (linha azul).

Figura 3 - Massa de água coletada na proveta durante a realização do ensaio com o comprimento da fôrma na posição vertical.

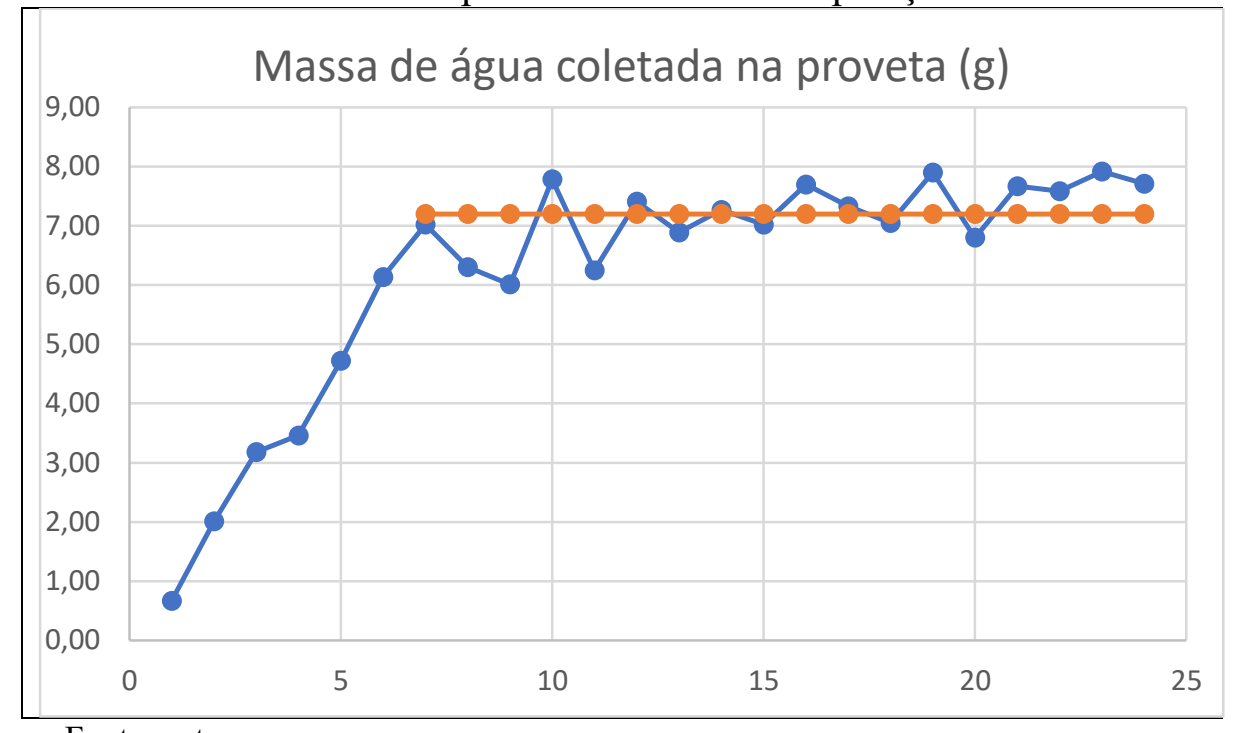

Fonte: autores

A situação correspondente ao ensaio no qual a altura da placa corresponde à largura da fôrma metálica produz um gráfico muito similar àquele apresentado na Figura 3, ressalvandose o fato de que a massa média de água coletada na proveta é de 7,31 gramas, valor superior ao outro ensaio. Tal fato condiz com o modelo adotado para avaliar o processo de transferência de calor na fusão da placa de gelo, conforme será visto na seção seguinte. Esse segundo ensaio teve a duração de 33 minutos e a oscilação no valor da massa de água coletada na proveta iniciou-se a partir dos 8 minutos.

\section{DISCUSSÃO DOS RESULTADOS}

Após a realização dos ensaios foram avaliadas, com o emprego do conjunto de equações detalhado na seção 2 , as parcelas de calor transferidas por radiação, convecção e condensação de vapor de água sobre a superfície da placa. O somatório dessas parcelas permite avaliar a quantidade de calor teórica transferida do ar ambiente para a placa de gelo a cada intervalo de um minuto, no ensaio realizado. A quantidade de água derretida que foi coletada na proveta permite relacionar a real quantidade de calor absorvida pela placa de gelo. O valor correspondente ao coeficiente de emissividade da superfície da placa de gelo foi estimado em 0,97, com base nos dados consultados em literatura científica (SIEGE; HOWELL, 1992).

$\mathrm{Na}$ Tabela 1 são confrontados os dados experimentais e teóricos relativos ao calor transferido entre o ar ambiente e a placa de gelo no oitavo minuto de ensaio, os quais estão relacionados nas colunas denotadas por "Q fusão real" e "Q teor". As duas linhas indicadas 
apresentam os dados relativos às situações em que o o comprimento da fôrma corresponde à altura e à largura da placa de gelo; isto é, posição vertical e horizontal, respectivamente. Nessa tabela também são indicados os valores das parcelas de radiação, condensação e convecção, que integram o calor total teórico transferido para a placa de gelo, identificadas por "Q rad", "Q cond" e "Q conv", respectivamente.

Tabela 1 - Dados relativos aos dois ensaios, no intervalo correspondente ao oitavo minuto.

\begin{tabular}{|c|c|c|c|c|c|c|c|c|}
\hline Posição & $\begin{array}{c}\text { massa } \\
\text { gelo } \\
(\mathrm{g})\end{array}$ & $\begin{array}{c}\text { Q fusão } \\
(\mathrm{J})\end{array}$ & $\begin{array}{c}\text { Q rad } \\
(\mathrm{J})\end{array}$ & $\begin{array}{c}\text { Q conv } \\
(\mathrm{J})\end{array}$ & $\begin{array}{c}\text { Q cond } \\
(\mathrm{J})\end{array}$ & $\begin{array}{c}\text { Q teor } \\
(\mathrm{J})\end{array}$ & $\begin{array}{c}\text { Q fusão } \\
\text { real } \\
(\mathrm{J})\end{array}$ & $\begin{array}{c}\text { Erro } \\
\text { relativo } \\
(\%)\end{array}$ \\
\hline Vertical & 6,30 & 2106,7 & 771,67 & 697,99 & 778,86 & 2248,5 & 1991,33 & 11,44 \\
\hline Horizontal & 6,89 & 2305,0 & 771,67 & 775,82 & 865,71 & 2413,2 & 2176,77 & 9,80 \\
\hline
\end{tabular}

Fonte: autores

Cabe registrar que no ensaio em que o comprimento da fôrma corresponde à altura da placa, o erro médio relativo foi de $7,25 \%$, considerando os valores registrados do sétimo minuto até o final do ensaio, sendo que tal erro oscila entre 0,75 e $15,75 \%$. Na situação em que o comprimento da fôrma corresponde à largura da placa, o erro relativo médio foi de $7,94 \%$, com valores que oscilaram entre 0,24 e $20,87 \%$.

Confrontando os dados da Tabela 1, percebe-se que as parcelas teóricas correspondentes ao calor transferido por convecção e condensação de vapor de água na superfície da placa são distintas nas duas situações indicadas. Isso ocorre devido ao fato de que os coeficientes convectivos de transferência de calor e massa variam com o comprimento característico da superfície na qual ocorre a convecção natural, no caso, a altura da placa de gelo. Apenas a parcela de calor transferida por radiação é idêntica nas duas tabelas, pois essa parcela é função apenas à área da placa de gelo e de sua temperatura, não estando associada ao escoamento do fluido junto à placa. Desta forma, é possível associar ao valor do calor teórico absorvido pela placa de gelo uma massa de gelo derretida, de forma constante, ao longo de cada ensaio. Ao valor da massa calculada deve ser acrescida a massa de água que condensa na placa.

No ensaio em que a altura da placa é menor, a massa teórica de água coletada na proveta é de 7,60 gramas. Nesse caso, a massa experimental média coletada foi de 7,31 gramas, o que resulta num desvio de $3,82 \%$ entre o valor teórico e o valor experimental. No ensaio em que a altura da placa é maior, a massa teórica de água coletada na proveta é de 7,07 gramas. Nesse caso, a massa experimental média coletada foi de 7,20 gramas, o que resulta num desvio de $1,84 \%$ entre o valor teórico e o valor experimental.

\section{CONCLUSÕES}

A partir dos resultados teóricos e experimentais obtidos, podemos afirmar que uma placa retangular de gelo irá derreter mais rapidamente se o seu lado maior estiver na posição horizontal.

Os desvios médios absolutos verificados entre os valores teóricos e experimentais da massa de água coletada na proveta, em ambos os ensaios realizados, foram inferiores a $5 \%$; um valor que pode ser considerado pequeno para um experimento didático que requer apenas o emprego de uma balança analítica e um termohigrômetro como instrumentos de medição e não utiliza equipamento sofisticado ou de custo elevado.

São obtidos desvios ainda menores quando se confronta o valor teórico e o valor médio experimental da água coletada na proveta. Isso ocorre porque um dos principais fatores de erro no experimento realizado é a permanência de algumas gotículas de água na calha plástica 
que conduz o líquido que pinga da placa e se deposita na proveta. Assim, em alguns intervalos há uma maior quantidade de gotículas, ou gotículas de maior tamanho, aderidos na superfície interna da calha do que em outros intervalos, acentuando a oscilação da massa de água coletada.

Cabe aqui ainda enfatizar que a diferença relativa entre os valores teóricos para os dois tipos de ensaio foi de 7,22\%; o que pode ser considerado um valor baixo e que eventuais erros experimentais poderiam produzir resultados que não confirmassem as previsões teóricas associadas ao modelo proposto. Assim, o fato de se obter resultados compatíveis com as previsões do modelo - que indicam um desvio relativamente pequeno entre os dois tipos de ensaio - ressalta a aproximação quantitativa entre dados teóricos e experimentais, que pode ser considerada muita boa diante da simplicidade do material e dos instrumentos de medição utilizados no experimento. Destacamos também fato de que o experimento pode ser facilmente reproduzido em outras instituições de ensino superior, bastando para isso um estudo prévio teórico de alguns mecanismos de transferência de calor e massa, indicados na seção 2 deste artigo. Além disso, este experimento propicia um roteiro para o estudo experimental desse tema.

O estudo das equações associadas aos números adimensionais que, para muitos alunos, pode ser um assunto de pouco interesse, é apresentado sob a ótica de uma pergunta instigante: "uma placa de gelo retangular suspensa no ar poderá derreter mais rapidamente em função do seu posicionamento em relação ao eixo vertical? " ; sendo tal fato importante para manter o aluno motivado a observar e comparar as duas formas de realização do ensaio de fusão da placa, realizar a rotina de cálculos descrita no modelo matemático e confrontar dados quantitativos teóricos e experimentais.

Finalmente, conclui-se que o modelo teórico proposto, além de propiciar resultados quantitativos satisfatórios, com baixa discrepância entre dados experimentais e teóricos, demonstrou, através de um experimento de fácil execução, a influência do comprimento característico do escoamento num processo de convecção natural em que ocorre transferência simultânea de calor e massa.

\section{REFERÊNCIAS}

BEJAN, Adrian. Transferência de Calor, 570 pp. Ed. Edgard Blucher, São Paulo, 1996.

ÇENGEL, Yunus A.; GHAJAR, Afshin J. Transferência de Calor e Massa: Uma abordagem prática. Editora Mc-Graw Hill, Porto Alegre, 2012.

FOUST, Alan, S et al. Princípio das Operações Unitárias. Rio de Janeiro: Editora Guanabara Dois, 1982.

HOLMAN, Jack Phillip. Transferência de calor. Editora Mc-Graw Hill, São Paulo, 1983.

INCROPERA, Frank P.; DE WITT, David. Fundamentos de Transferência de Calor e Massa. Editora LTC, Rio de Janeiro, 2008.

KAYS, William M.; CRAWFORD, Michael E.; WEIGAND, Bernard. Convective Heat and Mass Transfer, Editora McGraw-Hill, 4a ed., 2004. 
MARCZAK, Lígia Damasceno Ferreira et al. Uma nova Metodologia para o ensino das disciplinas de Fenômenos de Transporte. In: XXXI COBENGE - Congressso Brasileiro de Engenharia. Anais. Rio de Janeiro, 2003.

MORAES, Roque. Construtivismo e Ensino de Ciências: Reflexões Epistemológicas e Metodológicas. Ed. EDIPUCRS, Porto Alegre, 2003.

SIEGEL, Robert; HOWELL, John R. Thermal Radiation Heat Transfer. Washington, 1992.

\title{
EXPERIMENTAL STUDY OF NATURAL HEAT CONVECTION ON A RECTANGULAR ICE SHEET
}

\begin{abstract}
The melting process of a rectangular ice sheet in quiescent air is used for the experimental study of the mechanisms of heat and mass transfer, and the dimensionless numbers involved in natural convection. Investigations about the spatial positioning of the ice plate on the fusion process is carried out in order to arouse the interest of students in the study of heat and mass transfer. As detailed in the text, convection and heat radiation, as well as the condensation of air humidity, are three heat transfer relevant mechanisms in melting ice. A mathematical model is proposed in order to estimate the heat amount associated with each mechanism Theoretical values are compared with experimental data and the quantitative results obtained were satisfactory, in accordance with theoretical predictions regarding the plate spatial positioning.
\end{abstract}

Keywords: Heat and mass transfer. Natural convection. Heat radiation. Low-cost didactic experiments. 